\title{
融合主曲率与颜色信息的彩色图像检索算法
}

\author{
程德强 ${ }^{1,2)}$, 张晧翔 ${ }^{1,2)}$, 江曼 1,2 , 寇旗旗 1,3$)^{*}$ \\ 1) (地下空间智能控制教育部工程研究中心 徐州 221116) \\ 2) (中国矿业大学信息与控制工程学院 徐州 221116) \\ 3) (中国矿业大学计算机科学与技术学院 徐州 221116) \\ (kouqiqi@cumt.edu.cn)
}

\begin{abstract}
摘 要: 针对当前图像检索算法存在特征维度高、检索耗时久、精度低等问题，提出一种基于颜色信息和纹理信息 相融合的图像检索算法. 首先对彩色图像进行颜色量化和统计以获取其颜色直方图, 利用海森矩阵提取彩色图像的 表面几何结构曲率信息，并根据提取的曲率信息构建方向梯度加权联合直方图; 然后对获得的颜色直方图和加权联 合方向梯度直方图进行融合, 得到最终检索结果. 与其他算法在 Corel-1k, FTVL 和 COIL-100 数据库上的实验结果表 明，该算法具有精度高、维度低的优点.
\end{abstract}

关键词：图像检索；颜色直方图；几何结构曲率信息；加权联合直方图

中图法分类号: TP391.41 DOI: 10.3724/SP.J.1089.2021.18406

\section{Color Image Retrieval Method Fusing Principal Curvature and Color Information}

\author{
Cheng Deqiang ${ }^{1,2)}$, Zhang Haoxiang ${ }^{1,2)}$, Jiang Man ${ }^{1,2)}$, and Kou Qiqi ${ }^{1,3)^{*}}$ \\ 1) (Engineering Research Center of Intelligent Control of Underground Space, Ministry of Education, Xuzhou 221116) \\ 2) (School of Information and Control Engineering, China University of Mining and Technology, Xuzhou 221116) \\ ${ }^{3)}$ (School of Computer Science and Technology, China University of Mining and Technology, Xuzhou 221116)
}

\begin{abstract}
Aiming at the problems of high feature dimensionality, long retrieval time, and low precision of existing algorithms in image retrieval system, an image retrieval algorithm based on the fusion of color information and texture information is proposed. Firstly, the color image is quantized and counted to obtain color histogram. Secondly, the Hessian matrix is used to extract curvature information of the color image, and the weighted joint histogram of oriented gradient based on curvature information is constructed. Then, the obtained color histogram and histogram of oriented gradient are fused to obtain the final joint histogram. And the experimental results on the Corel-1k, FTVL, and COIL-100 datasets show that the proposed algorithm has the advantages of high precision and low dimensionality.
\end{abstract}

Key words: image retrieval; color histogram; geometric curvature information; weighted joint histogram

随着多媒体信息与计算机视觉技术的飞速发 展，图像处理与模式识别技术为人类的生活和工 作带来了极大的便利. 伴随着图像处理与模式识
别技术的发展，图像检索技术自 20 世纪 70 年代以 来, 一直是研究的热门课题. 目前, 图像检索技术 已被广泛应用于搜索引擎、医学等 ${ }^{[1]}$ 领域中.

收稿日期：2020-05-27; 修回日期：2020-11-03. 基金项目：国家自然科学基金(51774281); 国家重点研发计划(2018YFC0808302). 程德强(1979-), 男, 博士, 教授, 博士生导师, CCF 会员, 主要研究方向为图像处理、机器视觉; 张皓翔(1994一), 男, 硕士研究生, 主要研究方向为模式识别; 江曼(1996-), 女, 硕士研究生, 主要研究方向为目标识别; 寇旗旗(1988一), 男, 博士, 讲师, 论文通讯 作者, 主要研究方向为模式识别. 
早期的图像检索算法主要是基于文本的图像 检索，利用文本信息对图像的特征进行匹配和检索. 随后各种基于内容的图像检索算法 (content-based image retrieval, CBIR)陆续被提出, CBIR 通过提取 图像的视觉内容信息, 如颜色、纹理、形状等, 对 视觉内容信息进行描述和处理, 再运用差异性度 量公式(如欧几里得度量等)进行差异性度量和判 定，从而在图像数据库中检索出与样本图像相似 的结果. Low $\mathrm{e}^{[2]}$ 提出了著名的尺度不变特征转换 (scale-invariant feature transform, SIFT)算法，通过 提取图像的局部特征信息并进行转换和描述，使 其具有良好的尺度和选择不变特性, 有效地提高 了其在图像检索数据库中的检索精度. 在 Low $\mathrm{e}^{[2]}$ 工作的基础上, Gao 等 ${ }^{[3]}$ 提出了基于 SIFT 关键点注 意力模型的图像检索算法, 并取得了较好的检索 结果. Wang 等 ${ }^{[4]}$ 提出基于 SIFT 特征融合的图像检 索算法，该算法是将原始 SIFT 特征进行过滤以篮 选出具有代表意义的 SIFT 特征, 并与颜色信息相 结合, 以进一步提高检索精度. 但是, 基于 SIFT 特征的图像检索算法常存在维度过高、检索耗时长 的缺点. 针对 SIFT 算法的不足, 基于梯度直方图 (histogram of oriented gradient, HOG)、颜色直方图 (color histogram, CH)、局部二值模式(local binary pattern, LBP) 等 ${ }^{[5-7]}$ 的方法也先后被提出, 并得到 不断改进和优化. Savakis 等 ${ }^{[8]}$ 将主成分分析后的 HOG 作为训练样本, 有效地减少了训练时间. 因 颜色信息不会被平移等因素影响，所以常用来表 示图像属性. 但是颜色信息无法描述出图像的颜 色空间分布，往往不单独使用. ElAlami ${ }^{[9]}$ 提取颜色 共现矩阵和图案像素之间的差异等颜色信息以及 纹理信息作为检索特征. Kundu 等 ${ }^{[10]}$ 使用非二次采 样 Contourlet 变换的多尺度几何分析计算出的统计 特征对数据库中的每个图像进行编码并用于检索. Somnugpong 等 ${ }^{[11]}$ 将颜色相关图和边缘方向直方图 进行融合，提取图像颜色信息和几何信息用于检 索. Dubey 等 ${ }^{[12]}$ 提取彩色图像的多通道 LBP 特征, 用加法器和解码器的 2 种模式组合多通道 LBP. Pavithra 等 ${ }^{[13]}$ 提出用颜色量化信息、空间纹理信息 以及形状信息的组合作为图像检索特征集用于检 索. Ahmed 等 ${ }^{\left[{ }^{[14}\right]}$ 提出融合空间颜色信息与形状信 息形状，然后与兴趣点级联，采用词袋检索，通过 将局部图像形状特征和颜色空间信息进行特征融 合, 以提高其在复杂环境下的鲁棒性. 可以发现以 上算法是将颜色信息和纹理信息等作为特征集或
者特征组合用于检索，达到了不错的效果. 此外, 孙奇平 ${ }^{[15]}$ 提出基于卷积神经网络的检索算法, 其 用卷积神经网络作为特征提取, 并利用主成分分 析进行降维, 提高检索效率. 虽然已经提出了大量 的图像特征提取和图像检索算法, 并取得一定的 效果，但是现有的图像检索算法仍然在计算复杂 度、检索耗时和精度方面有待提高.

针对上述问题，本文提出一种基于特征融合 的图像检索算法, 分别提取图像的颜色信息和几 何结构曲率信息进行统计和描述; 然后通过多层 的方式融合上述图像信息, 既提高了检索精度, 还 降低了描述符的特征维度.

\section{1 相关理论}

\section{1 主曲率原理}

对于主曲率信息的空间结构特性的探索，Kou 等 ${ }^{[16]}$ 进行了深人的研究. 在二维平面空间中, 曲 率是指曲线上一点处的切线方向角对弧长的转动 率, 它反映了几何体的弯曲程度. 在三维欧几里得 空间中, 主曲率是指过可微曲面上 $p_{0}$ 点处存在唯 一的一个法向量和无穷多个曲面的法截面, 而每 一个法截面与曲面的交线在 $p_{0}$ 点的曲率称为法曲 率. 因此, 过 $p_{0}$ 点的无数个法截面就对应着无数个 法曲率, 所有法曲率中的最大值曲率和最小值曲 率统称为可微曲面上 $p_{0}$ 点的主曲率(principal curvatures, PCs). 最大值曲率 $\lambda_{1}$ 对应曲面的弯曲程度 大；同理，弯曲程度最小的曲面所对应的曲率 $\lambda_{2}$ 最小. 图像某点的主曲率如图 1 所示. 无论图像以 任意角度发生旋转变化, 该点的主曲率大小都会 保持不变 ${ }^{[16]}$, 说明利用主曲率信息来表示图像的 特征信息可以取得连续旋转不变的特性.

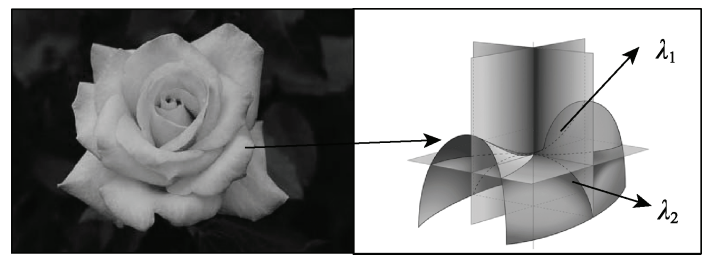

图 1 图像某点的主曲率示意图

\subsection{HOG 算法回顾}

自 $\mathrm{HOG}$ 特征描述符被提出以来，由于其具有 计算简单, 且对图像几何形变能保持很好的不变 性的优点, 已被广泛应用于图像识别领域的行人 
检测中. HOG 的中心思想是通过计算和统计图像 局部区域的梯度方向直方图描述图像特征，它将 图像分成小元胞; 然后采集元胞中各像素点梯度 或边缘方向直方图, 并将这些局部直方图在图像 更大的范围内(像素块)进行对比度归一化; 最后把 这些直方图组合起来就可以构成特征描述器.

计算图像中像素点的梯度信息分别为

$$
\begin{aligned}
G_{x}(x, y) & =H(x+1, y)-H(x-1, y), \\
G_{y}(x, y) & =H(x, y+1)-H(x, y-1) .
\end{aligned}
$$

其中, $G_{x}(x, y), G_{y}(x, y)$ 和 $H(x, y)$ 分别表示在像 素点 $(x, y)$ 处 $x$ 方向梯度, $y$ 方向梯度和灰度值, 则在该像素点处的梯度幅值和方向为

$$
\begin{aligned}
& G(x, y)=\sqrt{G_{x}(x, y)^{2}+G_{y}(x, y)^{2}}, \\
& \theta(x, y)=\arctan \left(\frac{G_{y}(x, y)}{G_{x}(x, y)}\right) .
\end{aligned}
$$

\section{2 本文算法}

本文首先在分析图像颜色信息的基础上构建 了基于 $\mathrm{HSV}$ 的 $\mathrm{CH}$ 模型; 其次，通过提取图像主曲 率信息提出加权方向梯度联合直方图 (weighted joint histogram of oriented gradient, WJHOG)的特 征描述子来统计图像主曲率信息，进而得到基于 主曲率的图像纹理信息直方图 (weighted joint histogram of oriented gradient based PCs, PC-WJHOG), 最后将颜色信息直方图和纹理信息直方图加权得 到基于合并的方向梯度特征融合直方图(fusion of incorporated HOG, F-IHOG). 本文算法的框架如图 2 所示.

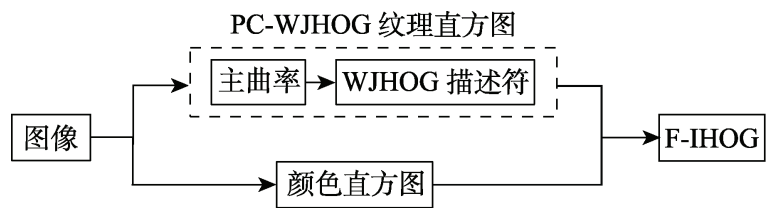

图 2 本文算法框架

\section{$2.1 \mathrm{CH}$ 构造}

作为一种有效的图像特征, $\mathrm{CH}$ 因具有平移、 缩放等特性而被广泛应用到计算机视觉领域中. 在构建之前, 首先将图像从 RGB 颜色空间转化为 HSV 颜色空间, 以获得更直观的特性, 然后对转 换后的 HSV 颜色信息进行空间量化以最小视觉失 真呈现原图像的空间颜色分布.

本文利用

$$
\begin{gathered}
h= \begin{cases}0, & H \in[0,20] \\
0, & H \in(315,360] \\
1, & H \in(20,40] \\
2, & H \in(40,75] \\
3, & H \in(75,155] \\
4, & H \in(155,190] \\
5, & H \in(190,270] \\
6, & H \in(270,295] \\
7, & H \in(295,315]\end{cases} \\
S= \begin{cases}0, & S \in[0,0.2] \\
1, & S \in(0.2,0.7] \\
2, & S \in(0.7,1]\end{cases} \\
v= \begin{cases}0, & V \in[0,0.2] \\
1, & V \in(0.2,0.7] \\
2, & V \in(0.7,1]\end{cases}
\end{gathered}
$$

对 HSV 颜色信息空间的 $H, S, V$ 通道进行量化; 然后用 $\boldsymbol{T}_{\mathrm{HSV}}=Q_{h} \times h+Q_{s} \times s+Q_{v} \times v$ 构造颜色直方 图. 其中, $Q_{h}=9, Q_{s}=3, Q_{v}=1 ; \boldsymbol{T}_{\mathrm{HSV}}$ 维度的取值 范围 $[0,1,2,3, \cdots, 71]$, 共 72 维. 实验发现, 直方图 最后一个分量所提供的颜色信息常为零, 因此本 文最终构建颜色信息 $\boldsymbol{T}_{\mathrm{HSV}}$ 维度为 71 维.

\subsection{PC-WJHOG 生成}

联合直方图融人了图像的主曲率信息以及方 向梯度信息, 先利用海森矩阵计算图像的表面几 何结构曲率信息中的主曲率信息，然后用方向梯 度联合直方图来统计所有像素点的主曲率信息, 进而得到 PC-WJHOG.

\subsection{1 图像主曲率信息}

计算主曲率信息之前，首先将图像灰度化; 然后构造灰度图像各像素点的海森矩阵; 接着根 据矩阵的行列式以及迹计算矩阵特征值以提取表 面几何结构曲率信息; 最后针对图像表面几何曲 率信息的特点, 对其转换和量化.

海森矩阵是一个多元函数的二阶偏导数构成 的方阵, 可用于描述函数的局部曲率. 灰度图像 I 在像素点 $(x, y)$ 处的海森矩阵可表示为

$$
\boldsymbol{H}(x, y)=\left|\begin{array}{cc}
D_{x x} & D_{x y} \\
D_{x y} & D_{y y}
\end{array}\right|=\left|\begin{array}{ll}
\boldsymbol{I} * \boldsymbol{G}_{x x} & \boldsymbol{I} * \boldsymbol{G}_{x y} \\
\boldsymbol{I} * \boldsymbol{G}_{x y} & \boldsymbol{I} * \boldsymbol{G}_{y y}
\end{array}\right| .
$$

其中, $*$ 表示卷积; $\boldsymbol{G}_{x x}, \boldsymbol{G}_{y y}$ 和 $\boldsymbol{G}_{x y}$ 分别为 $x, y$ 和 $x y$ 方向上的二阶导数卷积核. 3 个卷积核可以通过 对高斯函数进行二阶求导得到, 其求导结果分别为

$$
\boldsymbol{G}_{x x}=\frac{1}{2 \pi \sigma^{4}}\left(\frac{x^{2}}{\sigma^{2}}-1\right) \mathrm{e}^{-\frac{x^{2}+y^{2}}{2 \sigma^{2}}},
$$




$$
\begin{aligned}
& \boldsymbol{G}_{x y}=\frac{x y}{2 \pi \sigma^{6}} \mathrm{e}^{-\frac{x^{2}+y^{2}}{2 \sigma^{2}}}, \\
& \boldsymbol{G}_{y y}=\frac{1}{2 \pi \sigma^{4}}\left(\frac{y^{2}}{\sigma^{2}}-1\right) \mathrm{e}^{-\frac{x^{2}+y^{2}}{2 \sigma^{2}}} .
\end{aligned}
$$

其中, $\sigma$ 为高斯标准偏差. 矩阵特征值之积等于矩 阵的行列式, 矩阵的特征值之和等于矩阵的迹, 即

$$
\operatorname{tr} \boldsymbol{H}=\sum \lambda_{i}, \operatorname{det} \boldsymbol{H}=\prod \lambda_{i} .
$$

海森矩阵的 2 个特征值为

$$
\left\{\begin{array}{l}
\lambda_{1}=\frac{\operatorname{tr} \boldsymbol{H}+\sqrt{(\operatorname{tr} \boldsymbol{H})^{2}-4 \operatorname{det} \boldsymbol{H}}}{2} \\
\lambda_{2}=\frac{\operatorname{tr} \boldsymbol{H}-\sqrt{(\operatorname{tr} \boldsymbol{H})^{2}-4 \operatorname{det} \boldsymbol{H}}}{2} .
\end{array}\right.
$$

其中, $\lambda_{1}$ 和 $\lambda_{2}$ 均为像素点 $(x, y)$ 处的海森矩阵特征 值, 也称作 PCs.

PCs 描述了纹理信息的局部极值，反映了图像 的宏观信息和微观信息. 因此，PCs 不仅能够同时 表示图像的宏观和微观信息，还具有连续旋转不 变的特性. 图像在不同高斯标准偏差 $\sigma$ 下极值曲 率表示的结果如图 3 所示.

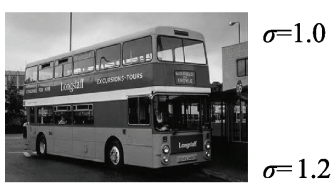

a. 灰度图

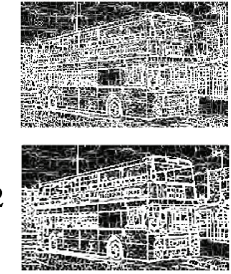

b. 极大曲率

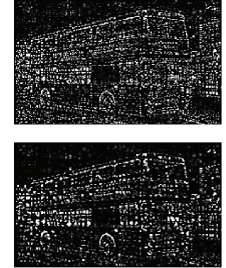

c. 极小曲率
图 3 主曲率表示的结果

为了增加前景与背景之间的对比度，同时便 于计算和统计基于主曲率信息的方向梯度直方图, 本文对主曲率进行操作

$$
\xi=\frac{\lambda_{1}}{\lambda_{2}}
$$

由于 $\xi$ 值比较小, 且在零附近小范围波动, 为 了提高区分度, 对 $\xi$ 进行幂指数转换, 即

$$
\eta=\frac{2}{1+\mathrm{e}^{(-2 \times \xi)}}-1
$$

用式(4)(5)对 $I(x, y)$ 中各像素点的主曲率信息 进行转换和量化之后，再利用第 2.2 .2 节中的 WJHOG 对 $\eta$ 进行统计.

\subsubsection{WJHOG 构造}

与 $\mathrm{HOG}$ 类似, WJHOG 先计算图像每个像素的 梯度(包括大小和方向). 如图 4 所示, WJHOG 的构 造步骤如下:
Step1. 将图像划分成小元胞.

Step2. 统计每个元胞的梯度直方图.

Step3. 每几个元胞组成一个像素块.

Step4. 以加权联合的方式将 $m \times n$ 个像素块进行累 加计算, 获得最终直方图.

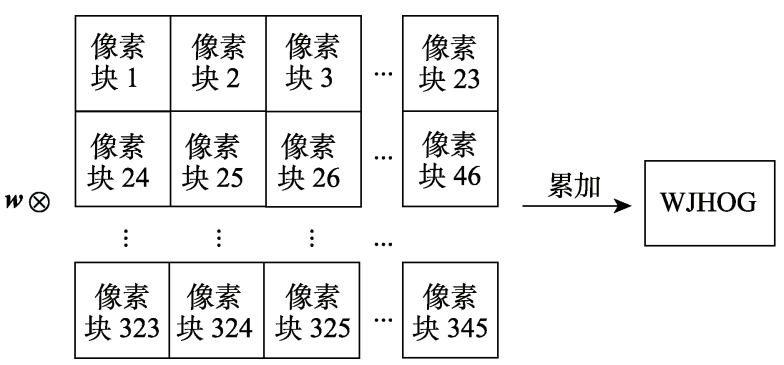

图 4 WJHOG 构造示意图

图 4 中, $\otimes$ 代表 $\boldsymbol{w}$ 与像素块对应相乘, $\boldsymbol{w}$ 为权 重矩阵，即

$$
\boldsymbol{w}=\left[\begin{array}{rrrrr}
0 & 0 & \cdots & 0 & 0 \\
0 & 1.05 & \cdots & 1.05 & 0 \\
\vdots & \vdots & & \vdots & \vdots \\
0 & 1.05 & \cdots & 1.05 & 0 \\
0 & 0 & \cdots & 0 & 0
\end{array}\right]_{15 \times 23} .
$$

WJHOG 将图像划分成小元胞, 每个元胞有 $8 \times 8$ 个像素点, 一个像素块包含 $4 \times 4$ 元胞, 即 $32 \times 32$ 个像素点, 每个元胞提供 9 维的 $\mathrm{HOG}$ 特征, 因此每个像素块可获得 144 维的 $\mathrm{HOG}$ 特征描述符. 以尺寸 $256 \times 384$ 的图像为例，按照上述处理方法， 并设置步长(step size, SS)为 16; 像素块尺寸(block size, BS)为 32, 将图像宽高代人

$$
B_{\text {number }}=\left(\left\lfloor\frac{I_{\mathrm{w}}-\mathrm{BS}}{\mathrm{SS}}\right\rfloor+1\right) \times\left(\left\lfloor\frac{I_{\mathrm{h}}-\mathrm{BS}}{\mathrm{SS}}\right\rfloor+1\right) \text {, }
$$

得到 $15 \times 23$ 个像素块. 其中, $I_{\mathrm{w}}$ 和 $I_{\mathrm{h}}$ 分别为图像 $\boldsymbol{I}(x, y)$ 的宽和高, $B_{\text {number }}$ 为像素块个数. 接着对 345 个像素块信息加权，与 $\mathrm{HOG}$ 不同的是， WJHOG 没有把像素块信息进行串联，而是把 345 个像素块进行叠加, 然后得到一个像素块, 该像素 块包含了 345 个像素块信息，因此可用于描述图像 特征, 这种方式使 WJHOG 描述符的特征长度仅为 144 维. 对第 2.2 .1 节中的 $\eta$ 计算和统计可得到 PC-WJHOG.

\subsection{F-IHOG 分析}

\subsection{1 特征融合}

颜色信息直观地反映了图像区域的表面性质, 且计算代价较小, 具有图像平移不变等优点, 主曲 率信息属于图像的纹理信息，不仅能够反映图像 的宏观和微观特征，且对光照条件的变化不敏感. 
因此，本文加权融合了颜色直方图和主曲率纹理 直方图，使图像检索描述符兼具颜色信息以及纹 理信息，即 $\boldsymbol{F}=w_{1} \boldsymbol{T}_{\mathrm{HSV}}+w_{2} \boldsymbol{P}_{\mathrm{PCs}}$. 其中, $\boldsymbol{T}_{\mathrm{HSV}}$ 和 $\boldsymbol{P}_{\mathrm{PCs}}$ 分别表示颜色信息和纹理信息; $w_{1}$ 和 $w_{2}$ 分别 为 $\boldsymbol{T}_{\mathrm{HSV}}$ 和 $\boldsymbol{P}_{\mathrm{PCs}}$ 权值.

\subsection{2 复杂度分析}

本文算法融合了基于 HSV 的 $\mathrm{CH}$ 模型和基于 主曲率的 PC-WJHOG 模型. 首先, 根据图像的颜 色分布构造 HSV 模型. 由于原始直方图维度过高, 不利于检索，为了减少模型的计算复杂度，对 HSV 的每个通道进行量化. 经式(1)(2)(3)量化后, 直方图维度大大降低且计算简单、高效. 在提取 PC-WJHOG 的过程中, 首先求得 $x, y$ 和 $x y$ 方向上 的二阶导, 为了降低主曲率计算过程的复杂度, 本 文构造了海森矩阵，根据矩阵的行列式和迹计算 矩阵的特征值, 求得各点主曲率, 达到降低复杂度 的目的. 进一步分析, CH 和 PC-WJHOG 的时间复 杂度分别为 $O\left(d_{1} \times M \times N\right)$ 和 $O\left(d_{2} \times M \times N\right)$. 其中, $M \times N$ 为图像尺寸; $d_{1}$ 为 $\mathrm{HSV}$ 通道数, $d_{1}=3 ; d_{2}$ 为海森矩阵尺寸, 这里 $d_{2}$ 为 $2 \times 2$.

\section{3 实验分析}

为了对本文图像检索算法的性能进行测试和 评估, 将各个算法在数据库 Corel- $1 \mathrm{k}^{[17]}$ 进行对比 实验. 此外, 本文选择在 FTVL ${ }^{[18]}$ 和 COIL-100 $0^{[19]}$ 数据库上进行光照、视角旋转变化测试, 并将测试 结果与其他在数据库上的主流算法的结果进行对 比和分析. 数据库中采用的度量方法为欧几里得度 量. 本文算法参数设置为 $\sigma=2, w_{1}=0.6, w_{2}=0.4$.

\section{1 数据库与实验设置}

在 Corel-1k 数据库中, 共有 10 类彩色图像, 分别为 Building, Flower, Africa, Horse, Mountain, Sea, Dinosaur, Bus, Elephant 和 Food, 如图 5 所示. 其中, 每类图像又包含 100 个样本, 所以 Corel-1k 数据库中共有 1000 幅图像.

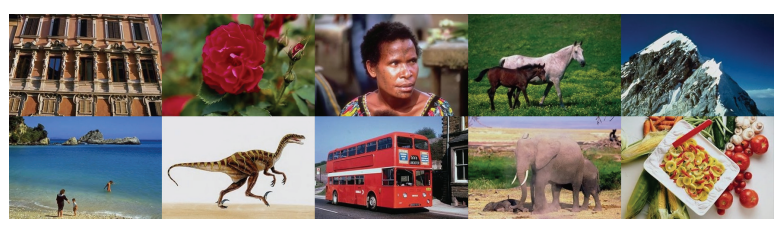

图 5 Corel-1k 样本图像

FTVL 数据库包含 2633 幅水果和蔬菜的图像, 按照种类的不同划分为图 6 所示 15 种, 分别是
Agata Potato, Asterix Potato, Cashew, Diamond Peach, Fuji Apple, Smith Apple, Melon, Kiwi, Nectarine, Onion, Orange, Plum, Spanish Pear, Taiti Lime 和 Watermelon. FTVL 数据库具有光照、视角 等一些变化特点.

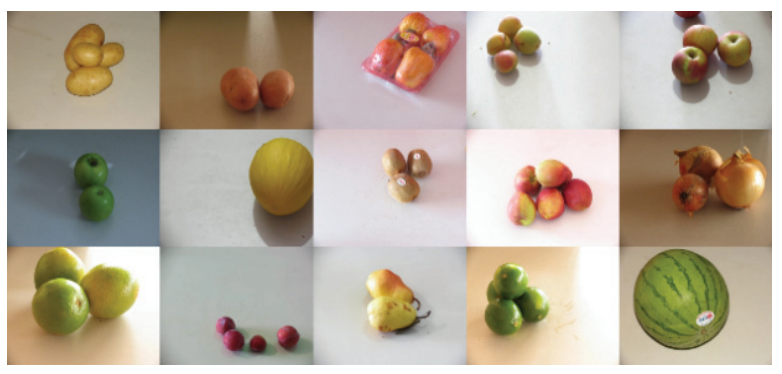

图 6 FTVL 样本图像

COIL-100 数据库中包含 100 幅不同类别的图 像，每类图像又包括同一种物品在不同角度下的 成像结果. 每种物品成像的旋转角度间隔为 $5^{\circ}$, 所 以在 $0^{\circ} \sim 360^{\circ}$ 范围内每种物品共有 72 个不同视角 下的成像结果. 因此, COIL-100 数据库共有 7200 幅图像, 且每幅图像均以黑色为背景. 不同视角下 的同一物品成像结果也大不相同, 所以对 COIL100 数据库中的图像进行高效精准的检索存在一 定难度. 图 7列举了 15 种类别的样本图像, 分别为 Tomato, Cat, White Cup, Frog, Mud Pot, Pink Cup, Fancy, Rolaids, Car, Soft Edri, Statue, Truck, Herb-0x, Feast Jug 和 Stick.

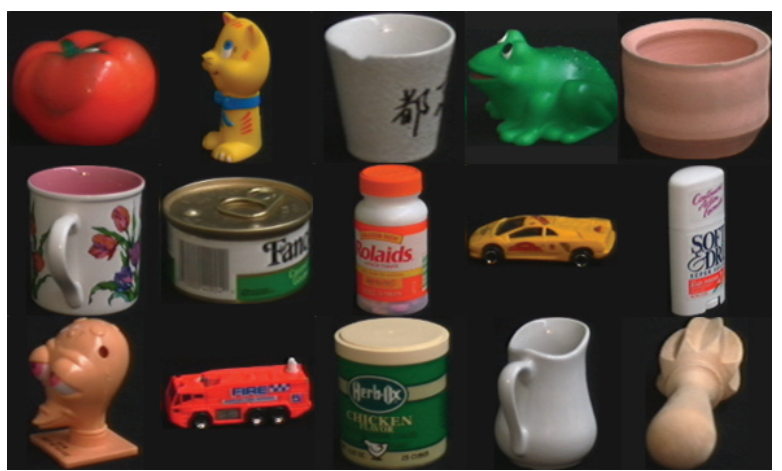

图 7 COIL-100 部分样本图像

\section{2 实验结果分析}

\section{2 .1 基于 $\mathrm{HOG}$ 算法实验对比}

为了验证本文的特征描述方法在特征维度方 面的优异表现, 选取了 HOG 和 PCA-HOG 算法作 为对比, 表 1 所示为各算法检索前 10 幅图像的平 均精度 (average precision, AP), 所有类别 AP 的均 值 (mean average precision, mAP), 特征维度 (dimension, Dim)和检索耗时 (retrieval time, RT). 
从表 1 可看出, 本文的 WJHOG, PC-WJHOG 和 F-IHOG 算法均优于传统 HOG 和 HOG-PCA. 其中, 检索类别为 Flower 时, PC-WJHOG 的检索精度要 比 HOG 算法检索精度高出 $53.0 \%$, F-IHOG 的检索 精度比 HOG 高出 $58.0 \%$, 说明基于融合的检索算
法优于单特征检索算法. WJHOG 算法的单幅图像 特征提取耗时(time for single feature extraction, SFET)为 $0.005 \mathrm{~s}$. F-IHOG 算法的 SFET 也仅为 $0.019 \mathrm{~s}$, 特征维度仅为 215 维. 说明本文的特征描述方法具 有速度快、精度高的优点.

表 1 基于 HOG 算法的对比结果

\begin{tabular}{|c|c|c|c|c|c|c|c|c|c|}
\hline \multirow{2}{*}{ 算法 } & \multicolumn{7}{|c|}{$\mathrm{AP} / \%$} & \multirow{2}{*}{$\mathrm{SFET} / \mathrm{s}$} & \multirow{2}{*}{ Dim } \\
\hline & Africa & Sea & Bus & Elephant & Flower & Dinosaur & Mountain & & \\
\hline $\mathrm{HOG}^{[5]}$ & 35.0 & 50.0 & 55.0 & 45.0 & 40.0 & 65.0 & 30.0 & 0.022 & 49680 \\
\hline HOG-PCA ${ }^{[8]}$ & 25.0 & 15.0 & 10.0 & 60.0 & 10.0 & 15.0 & 12.0 & 0.031 & 199 \\
\hline WJHOG & 50.0 & 52.0 & 96.0 & 53.0 & 89.0 & 93.0 & 38.0 & 0.005 & 144 \\
\hline PC-WJHOG & 75.0 & 70.0 & 98.0 & 70.0 & 93.0 & 98.0 & 50.0 & 0.009 & 144 \\
\hline F-IHOG & 95.0 & 75.0 & 100.0 & 75.0 & 98.0 & 100.0 & 80.0 & 0.019 & 215 \\
\hline
\end{tabular}

\subsubsection{Corel-1k 数据库实验结果}

表 2 和表 3 分别给出了不同算法在 Corel-1k 上的检索精度和召回率. 与文献[14]算法相比，本 文算法在一些类别的检索精度有很大提升; 与文 献[13]算法相比，本文算法在多数检索任务中均获 得较高的检索精度，在 Africa 和 Food 类别的检索 任务中，本文算法的检索精度比文献[13]算法分别
高出 $10.5 \%, 13.2 \%$. 而且与基于深度学习的算法 ${ }^{[15]}$ 相比，本文算法表现优异，Dinosaur 类别的检索精 度提升约 $30.0 \%$. 表 4 的 mAP 对比结果也表明，本 文的图像检索算法有较高的检索精度.从效率来 看，本文算法 SFET 仅为 $0.019 \mathrm{~s}$, RT 仅为 $0.641 \mathrm{~s}$. 分析可知, 本文算法在检索精度和效率方面均有 不错的表现.

表 2 不同算法在 Corel-1k 上的 AP

\begin{tabular}{|c|c|c|c|c|c|c|c|c|c|c|}
\hline 算法 & Africa & Sea & Building & Bus & Dinosaur & Elephant & Flower & Horse & Mountain & Food \\
\hline Pavithra 等 ${ }^{[13]}$ & 81.0 & 66.0 & 78.8 & 96.3 & 100.0 & 70.8 & 95.8 & 98.8 & 67.8 & 77.3 \\
\hline Ahmed 等 ${ }^{[14]}$ & 90.0 & 60.0 & 90.0 & 75.0 & 100.0 & 70.0 & 90.0 & 100.0 & 70.0 & 90.0 \\
\hline 孙奇平 ${ }^{[15]}$ & 33.0 & 22.0 & 40.0 & 23.3 & 71.0 & 27.5 & 50.0 & 59.2 & 26.7 & 65.0 \\
\hline Kundu 等 ${ }^{[10]}$ & 44.0 & 32.0 & 52.0 & 62.0 & 40.0 & 80.0 & 57.0 & 75.0 & 57.0 & 56.0 \\
\hline Somnugpong 等 ${ }^{[11]}$ & 67.6 & 59.8 & 58.0 & 94.0 & 99.8 & 58.0 & 88.6 & 93.8 & 47.8 & 49.2 \\
\hline Dubey 等 ${ }^{[12]}$ & 75.0 & 55.0 & 67.0 & 95.0 & 97.0 & 63.0 & 93.0 & 89.0 & 45.0 & 70.0 \\
\hline Xiao 等 ${ }^{[20]}$ & 67.0 & 60.0 & 56.0 & 96.0 & 98.0 & 53.0 & 93.0 & 82.0 & 46.0 & 58.0 \\
\hline Walia 等 ${ }^{[21]}$ & 73.0 & 39.2 & 46.3 & 82.5 & 98.0 & 59.3 & 86.0 & 89.8 & 41.8 & 53.0 \\
\hline Rao 等 ${ }^{[22]}$ & 56.0 & 53.0 & 61.0 & 89.0 & 98.0 & 57.0 & 89.0 & 78.0 & 51.0 & 69.0 \\
\hline 本文 & 91.5 & 67.0 & 70.5 & 98.5 & 100.0 & 69.0 & 96.0 & 100.0 & 73.0 & 90.5 \\
\hline
\end{tabular}

表 3 不同算法在 Corel-1k 上的召回率

\begin{tabular}{|c|c|c|c|c|c|c|c|c|c|c|}
\hline 算法 & Africa & Sea & Building & Bus & Dinosaur & Elephant & Flower & Horse & Mountain & Food \\
\hline Pavithra 等 ${ }^{[13]}$ & 16.2 & 13.2 & 15.8 & 19.3 & 20.0 & 14.2 & 19.2 & 19.8 & 13.6 & 15.5 \\
\hline Ahmed 等[14] & 18.0 & 12.0 & 18.0 & 15.0 & 20.0 & 14.0 & 18.0 & 20.0 & 14.0 & 18.0 \\
\hline 孙奇平[15] & 6.6 & 4.4 & 2.0 & 4.6 & 14.2 & 5.4 & 10.0 & 11.8 & 5.3 & 13.0 \\
\hline Kundu 等 ${ }^{[10]}$ & 8.8 & 6.4 & 10.4 & 12.4 & 8.0 & 16.0 & 11.4 & 15.0 & 11.4 & 11.2 \\
\hline Somnugpong 等 ${ }^{[11]}$ & 13.5 & 12.0 & 11.6 & 18.8 & 20.0 & 11.6 & 17.7 & 18.8 & 9.6 & 9.8 \\
\hline Dubey 等 ${ }^{[12]}$ & 15.0 & 11.0 & 13.4 & 19.0 & 19.4 & 12.6 & 18.6 & 17.8 & 9.0 & 14.0 \\
\hline $\mathrm{Xiao}$ 等 ${ }^{[20]}$ & 13.4 & 12.0 & 11.2 & 19.2 & 19.6 & 10.6 & 18.6 & 16.4 & 9.2 & 11.6 \\
\hline Walia 等[21] & 14.6 & 7.8 & 9.3 & 16.5 & 19.6 & 11.9 & 17.2 & 18.0 & 8.4 & 10.6 \\
\hline $\mathrm{Rao}$ 等 ${ }^{[22]}$ & 11.2 & 10.6 & 12.2 & 17.8 & 19.6 & 11.4 & 17.8 & 15.6 & 10.2 & 13.8 \\
\hline 本文 & 18.3 & 13.4 & 14.1 & 19.7 & 20.0 & 13.8 & 19.2 & 20.0 & 14.6 & 18.1 \\
\hline
\end{tabular}


表 4 不同算法在 Corel-1k 上的各项性能

\begin{tabular}{|c|c|c|c|c|}
\hline 算法 & $\mathrm{SFET} / \mathrm{s}$ & $\mathrm{RT} / \mathrm{s}$ & Dim & $\mathrm{mAP} / \%$ \\
\hline Pavithra 等 ${ }^{[13]}$ & 0.671 & 1.108 & $>768$ & 83.260 \\
\hline Ahmed 等 ${ }^{[14]}$ & 0.142 & 1.190 & & 83.500 \\
\hline 孙奇平[15] & 9.150 & 1.027 & 900 & 41.770 \\
\hline Kundu 等 ${ }^{[10]}$ & 0.400 & & 99 & 55.500 \\
\hline Somnugpong 等 ${ }^{[11]}$ & & & $>4096$ & 71.660 \\
\hline Dubey 等[12] & 102.400 & 16.490 & 1024 & 74.900 \\
\hline Xiao 等 ${ }^{[20]}$ & & & 63 & 70.100 \\
\hline Walia 等[21] & & & $>1240$ & 70.900 \\
\hline $\mathrm{Rao}$ 等 ${ }^{[22]}$ & 2.797 & 4.096 & 260 & 66.890 \\
\hline 本文 & 0.019 & 0.641 & 215 & 85.600 \\
\hline
\end{tabular}

\subsubsection{FTVL 数据库实验结果}

FTVL 数据库中的图像存在光照和视点角度 的变化, 为了测试本文算法在上述干扰条件下的
检索精度，将其与文献[18]提到的热带水果标准检 索算法 CCV+CLBP, CCV+LTP, CDH+SHE+CLBP 等算法的测试结果进行对比. 从表 5 可以看出，与 文献[14]算法相比，本文算法在 Fuji Apple，Watermelon 类别的检索结果高出 $6.0 \%$; 在 Orange, Asterix Potato, Watermelon 等类别的检索任务中, 本文检索精度达到 $100.0 \%$ 。其中，本文算法在 Orange 类别的检索任务中取得了最佳精度. 分析 上述实验可知, 本文算法在目标图像遭受光照、视 角方面干扰时，具有更好的鲁棒性.

\subsubsection{COIL-100 数据库实验结果}

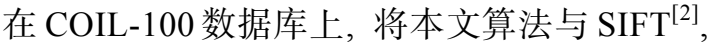
$\mathrm{LBP}^{[6]}, \mathrm{SURF}^{[23]}, \mathrm{MSER}^{[24]}$, DoG ${ }^{[25]}$ 等算法进行对 比, 实验结果如表 6 所示. 分析结果可知, 本文算 法在大多数类别均获得最佳检索精度，与文献[14]

表 5 不同算法在 FTVL 上的 AP

\begin{tabular}{|c|c|c|c|c|c|c|c|c|}
\hline 类别 & 本文 & Ahmed 等 ${ }^{[14]}$ & $\mathrm{CCV}+\mathrm{CLBP}^{[18]}$ & $\mathrm{CCV}+\mathrm{LTP}^{[18]}$ & $\mathrm{CDH}+\mathrm{SHE}+\mathrm{CLBP}^{[18]}$ & $\mathrm{GCH}+\mathrm{LBP}^{[18]}$ & $\mathrm{CDH}+\mathrm{SHE}^{[18]}$ & $\overline{\mathrm{CDH}+\mathrm{CLBP}^{[18]}}$ \\
\hline Agata Potato & 98.0 & 97.0 & 100.0 & 100.0 & 99.0 & 100.0 & 96.0 & 93.0 \\
\hline Asterix Potato & 100.0 & 100.0 & 98.0 & 99.0 & 96.0 & 96.0 & 96.0 & 95.0 \\
\hline Cashew & 100.0 & 99.0 & 100.0 & 100.0 & 100.0 & 100.0 & 100.0 & 100.0 \\
\hline Diamond Peach & 85.0 & 91.0 & 100.0 & 100.0 & 96.0 & 100.0 & 90.0 & 96.0 \\
\hline Fuji Apple & 99.0 & 93.0 & 63.0 & 67.0 & 71.0 & 75.0 & 60.0 & 59.0 \\
\hline Smith Apple & 100.0 & 97.0 & 100.0 & 100.0 & 99.0 & 100.0 & 96.0 & 97.0 \\
\hline Melon & 92.0 & 94.0 & 99.0 & 99.0 & 98.0 & 99.0 & 90.0 & 99.0 \\
\hline Kiwi & 98.0 & 95.0 & 70.0 & 75.0 & 77.0 & 70.0 & 76.0 & 75.0 \\
\hline Nectarine & 96.0 & 95.0 & 84.0 & 88.0 & 90.0 & 87.0 & 85.0 & 81.0 \\
\hline Onion & 90.0 & 89.0 & 100.0 & 100.0 & 100.0 & 100.0 & 99.0 & 100.0 \\
\hline Orange & 100.0 & 96.0 & 98.0 & 99.0 & 98.0 & 99.0 & 97.0 & 98.0 \\
\hline Plum & 92.0 & 90.0 & 98.0 & 100.0 & 99.0 & 99.0 & 98.0 & 97.0 \\
\hline Spanish Pear & 94.0 & 91.0 & 86.0 & 75.0 & 86.0 & 84.0 & 70.0 & 83.0 \\
\hline Taiti Lime & 100.0 & 100.0 & 100.0 & 100.0 & 99.0 & 100.0 & 96.0 & 100.0 \\
\hline Watermelon & 100.0 & 94.0 & 99.0 & 100.0 & 99.0 & 99.0 & 99.0 & 98.0 \\
\hline
\end{tabular}

表 6 不同算法在 COIL-100 上的 AP

\begin{tabular}{|c|c|c|c|c|c|c|c|c|}
\hline 类别 & 本文 & Ahmed 等 ${ }^{[14]}$ & SURF $^{[23]}$ & $\operatorname{RGBLBP}^{[7]}$ & $\operatorname{MSER}^{[24]}$ & $\operatorname{DoG}^{[25]}$ & $\mathrm{LBP}^{[6]}$ & $\mathrm{SIFT}^{[2]}$ \\
\hline Tomato & 98.0 & 93.0 & 75.0 & 20.0 & 15.0 & 60.0 & 35.0 & 15.0 \\
\hline Car & 97.0 & 98.0 & 65.0 & 55.0 & 22.0 & 15.0 & 60.0 & 22.0 \\
\hline Pink Cup & 100.0 & 88.0 & 50.0 & 50.0 & 35.0 & 30.0 & 60.0 & 40.0 \\
\hline Cat & 100.0 & 90.0 & 45.0 & 25.0 & 55.0 & 10.0 & 40.0 & 32.0 \\
\hline White Cup & 100.0 & 94.0 & 40.0 & 50.0 & 60.0 & 25.0 & 25.0 & 45.0 \\
\hline Truck & 85.0 & 90.0 & 35.0 & 60.0 & 35.0 & 20.0 & 30.0 & 15.0 \\
\hline Statue & 100.0 & 100.0 & 30.0 & 55.0 & 45.0 & 10.0 & 25.0 & 35.0 \\
\hline stick & 87.0 & 93.0 & 35.0 & 10.0 & 90.0 & 15.0 & 50.0 & 30.0 \\
\hline Soft Edri & 96.0 & 95.0 & 80.0 & 50.0 & 50.0 & 25.0 & 60.0 & 20.0 \\
\hline Rolaids & 100.0 & 65.0 & 60.0 & 85.0 & 40.0 & 50.0 & 65.0 & 20.0 \\
\hline Mud Pot & 100.0 & 100.0 & 45.0 & 50.0 & 90.0 & 50.0 & 70.0 & 20.0 \\
\hline Herb-0x & 100.0 & 90.0 & 45.0 & 65.0 & 25.0 & 20.0 & 40.0 & 25.0 \\
\hline Frog & 97.0 & 95.0 & 65.0 & 45.0 & 45.0 & 45.0 & 55.0 & 20.0 \\
\hline Fancy Feast & 100.0 & 100.0 & 20.0 & 45.0 & 35.0 & 40.0 & 60.0 & 25.0 \\
\hline Jug & 100.0 & 100.0 & 45.0 & 60.0 & 70.0 & 25.0 & 65.0 & 20.0 \\
\hline
\end{tabular}


算法实验结果相比，本文的 F-IHOG 描述符在类 别 Tomato, Pink Cup 和 Cat 上的检索精度分别提 升了 5.0\%, 12.0\%和 10.0\%; 其中, 在 Fancy Feast, Pink Cup 和 Cat 等类别的检索任务精度达到 $100.0 \%$. 由上述实验结果可知，与其他主流检索 算法相比，本文算法对于彩色图像视角变化具有 更高的鲁棒性.

\section{4 结 语}

本文提出了一种彩色图像检索算法 F-IHOG, 采用颜色直方图表示彩色图像颜色信息，利用海 森矩阵提取的图像表面曲率信息构建方向梯度加 权联合直方图表示图像纹理信息，最后将颜色信 息和纹理信息进行加权融合. 在 Corel-1k, FTVL, COIL-100 数据库上进行测试, 并将测试结果与当 前流行的算法进行对比. 结果表明，本文算法具有 较低的特征维度，因此在目标检索时具有较低的 计算耗时. 此外, 与当前流行的算法相比, 本文算 法对光照变化、视角变化方面的干扰具有更高的鲁 棒性，可以实现简单、高精度的图像检索. 但是, 本文算法对图像尺度变化的鲁棒性不高. 因此, 下 一步的工作重点主要是研究对尺度变化也具有不 变性的图像检索算法.

\section{参考文献(References):}

[1] Wu Menglin, Chen Qiang, Sun Quansen. Medical case retrieval based on combination of images and textual information[J]. Journal of Computer-Aided Design \& Computer Graphics, 2014, 26(9): 1430-1437(in Chinese)

(吴梦麟，陈强，孙权森. 结合影像和文本信息的医学病例 检索 $[J]$. 计算机辅助设计与图形学学报, 2014, 26(9): 14301437)

[2] Lowe D G. Distinctive image features from scale-invariant keypoints[J]. International Journal of Computer Vision, 2004, 60(2): 91-110

[3] Gao K, Lin S X, Zhang Y D, et al. Attention model based SIFT keypoints filtration for image retrieval[C] //Proceedings of the 7th IEEE/ACIS International Conference on Computer and Information Science. Los Alamitos: IEEE Computer Society Press, 2008: 191-196

[4] Wang Z Z, Jia K B, Liu P Y. A novel image retrieval algorithm based on ROI by using SIFT feature matching[C] //Proceedings of International Conference on Multimedia and Information Technology. Los Alamitos: IEEE Computer Society Press, 2008: 338-341

[5] Dalal N, Triggs B. Histograms of oriented gradients for human
detection[C] //Proceedings of the IEEE Computer Society Conference on Computer Vision and Pattern Recognition. Los Alamitos: IEEE Computer Society Press, 2005: 886-893

[6] Ojala T, Pietikainen M, Maenpaa T. Multiresolution gray-scale and rotation invariant texture classification with local binary patterns[J]. IEEE Transactions on Pattern Analysis and Machine Intelligence, 2002, 24(7): 971-987

[7] Ojala T, Pietikäinen M, Mäenpää T. Gray scale and rotation invariant texture classification with local binary patterns[C] // Proceedings of the European Conference on Computer Vision. Heidelberg: Springer, 2000: 404-420

[8] Savakis A, Sharma R, Kumar M. Efficient eye detection using HOG-PCA descriptor[C] //Proceedings of Imaging and Multimedia Analytics in a Web and Mobile World 2014. Bellingham: Society of Photo-Optical Instrumentation Engineers, 2014, 9027: 90270J

[9] ElAlami M E. A new matching strategy for content based image retrieval system[J]. Applied Soft Computing, 2014, 14: 407-418

[10] Kundu M K, Chowdhury M, Rota Bulò S. A graph-based relevance feedback mechanism in content-based image retrieval[J]. Knowledge-Based Systems, 2015, 73(1): 254-264

[11] Somnugpong S, Khiewwan K. Content-based image retrieval using a combination of color correlograms and edge direction histogram $[\mathrm{C}] / /$ Proceedings of the 13th International Joint Conference on Computer Science and Software Engineering. Los Alamitos: IEEE Computer Society Press, 2016: 417-421

[12] Dubey S R, Singh S K, Singh R K. Multichannel decoded local binary patterns for content-based image retrieval[J]. IEEE Transactions on Image Processing, 2016, 25(9): 4018-4032

[13] Pavithra L K, Sharmila T S. An efficient framework for image retrieval using color, texture and edge features[J]. Computers \& Electrical Engineering, 2018, 70: 580-593

[14] Ahmed K T, Ummesafi S, Iqbal A. Content based image retrieval using image features information fusion[J]. Information Fusion, 2019, 51: 76-99

[15] Sun Qiping. Research on image retrieval based on deep learning[J]. Journal of Jingdezhen University, 2018, 33(3): 15-18(in Chinese)

(孙奇平. 基于深度学习的图像检索研究 [J]. 景德镇学院学 报, 2018, 33(3): 15-18)

[16] Kou Q Q, Cheng D Q, Chen L L, et al. A multiresolution gray-scale and rotation invariant descriptor for texture classification[J]. IEEE Access, 2018, 6: 30691-30701

[17] Wang J Z, Li J, Wiederhold G. SIMPLIcity: semantics-sensitive integrated matching for picture libraries[J]. IEEE Transactions on Pattern Analysis and Machine Intelligence, 2001, 23(9): 947-963

[18] Dubey S R, Jalal A S. Fruit and vegetable recognition by fusing colour and texture features of the image using machine learning $[\mathrm{J}]$. International Journal of Applied Pattern Recognition, 2015, 2(2): 160-181

[19] Nene S A, Nayar S K, Murase H. Object image library 
(COIL-100)[R]. Columbia: Columbia University. Department of Computer Science, 1996

[20] Xiao Y, Wu J X, Yuan J S. mCENTRIST: a multi-channel feature generation mechanism for scene categorization[J]. IEEE Transactions on Image Processing, 2014, 23(2): 823-836

[21] Walia E, Vesal S, Pal A. An effective and fast hybrid framework for color image retrieval[J]. Sensing and Imaging, 2014, 15(1): 93-55

[22] Rao M B, Rao B P, Govardhan A. CTDCIRS: content based image retrieval system based on dominant color and texture features[J]. International Journal of Computer Applications,
2011, 18(6): Article No.8

[23] Bay H, Ess A, Tuytelaars T, et al. Speeded-up robust features (SURF)[J]. Computer Vision and Image Understanding, 2008, 110(3): 346-359

[24] Matas J, Chum O, Urban M, et al. Robust wide-baseline stereo from maximally stable extremal regions[J]. Image and Vision Computing, 2004, 22(10): 761-767

[25] Xu X Y, Miller E L. Adaptive difference of Gaussians to improve subsurface imagery[C] //Proceedings of the IEEE International Conference on Image Processing. Los Alamitos: IEEE Computer Society Press, 2002: 3441-3443 